\title{
Millisecond radiative recombination in poly(phenylene vinylene)-based light-emitting diodes from transient electroluminescence
}

Cite as: J. Appl. Phys. 101, 114506 (2007); https://doi.org/10.1063/1.2743741

Submitted: 19 February 2007 . Accepted: 16 April 2007. Published Online: 11 June 2007

Germà Garcia-Belmonte, José M. Montero, Eva M. Barea, Juan Bisquert, Henk J. Bolink, et al.

\section{ARTICLES YOU MAY BE INTERESTED IN}

Transient and steady-state behavior of space charges in multilayer organic light-emitting diodes

Journal of Applied Physics 89, 4575 (2001); https://doi.org/10.1063/1.1352027

Electron mobility in tris(8-hydroxy-quinoline)aluminum thin films determined via transient electroluminescence from single- and multilayer organic light-emitting diodes Journal of Applied Physics 89, 3711 (2001); https://doi.org/10.1063/1.1330766

Transient electroluminescence dynamics in small molecular organic light-emitting diodes Applied Physics Letters 97, 113301 (2010); https://doi.org/10.1063/1.3481687

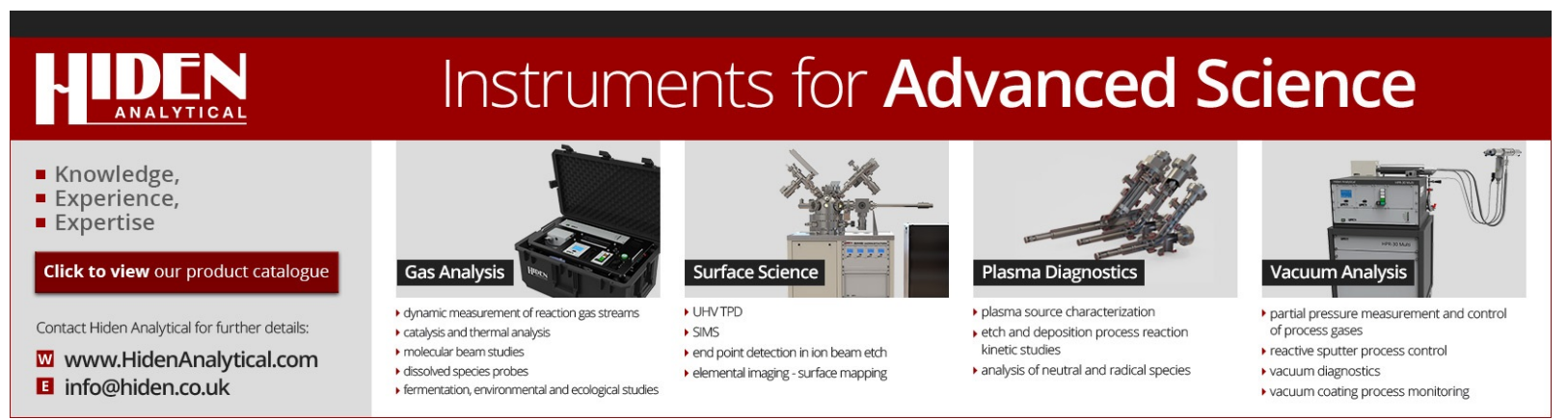




\title{
Millisecond radiative recombination in poly(phenylene vinylene)-based light-emitting diodes from transient electroluminescence
}

\author{
Germà Garcia-Belmonte, ${ }^{\text {a) }}$ José M. Montero, Eva M. Barea, and Juan Bisquert \\ Departament de Física, Universitat Jaume I, E-12071 Castelló, Spain \\ Henk J. Bolink \\ Institut de Ciència Molecular, Universitat de València, Polígon La Coma s/n, E-46980, Paterna, València, \\ Spain
}

(Received 19 February 2007; accepted 16 April 2007; published online 11 June 2007)

\begin{abstract}
The current and electroluminescence transient responses of standard poly(phenylene vinylene)-based light-emitting devices have been investigated. The electroluminescence time response is longer (milliseconds scale) than the current switch-off time by more than one order of magnitude, in the case of small area devices $\left(<0.1 \mathrm{~cm}^{2}\right)$. For large area devices $\left(\sim 6 \mathrm{~cm}^{2}\right)$ the electroluminescence decay time decreases from $1.45 \mathrm{~ms}$ to $\sim 100 \mu$ s with increasing bias voltage. The fast current decay limits the electroluminescence decay at higher voltages. Several approaches are discussed to interpret the observed slow decrease of electroluminescence after turning off the bias. One relies upon the Langevin-type bimolecular recombination kinetics which is governed by the minority carriers (electrons), and another focuses on the slow release of trapped electrons as possible explanations. Additionally, we show that the device current density is mainly determined by the transport of the fastest carriers (holes). (C) 2007 American Institute of Physics.
\end{abstract}

[DOI: $10.1063 / 1.2743741]$

\section{INTRODUCTION}

Transient electroluminescence (EL) of polymer- and small molecule-based light-emitting devices (OLEDs) has been the subject of intense technological as well as fundamental research, because the response time of these devices provides an essential criterion for their application. ${ }^{1-14} \mathrm{Be}$ sides the evident importance of achieving fast time responses for incorporating OLEDs as light sources for data communication systems, ${ }^{13}$ transient EL studies have generated insight into some of the physical parameters controlling the operation of these devices, in particular, device interfacial effects. For instance, some device structures exhibit an EL overshoot when the driving voltage is switched off, which was related to the delayed recombination of stored electrons and holes. ${ }^{5,10}$ In single-layer OLEDs a delayed onset, $t_{d}$, in the EL response with respect to the voltage turning on is often reported. ${ }^{1}$ This delay time was interpreted as being the transit time of the majority charge carriers, and is used to determine their carrier mobility. In other single- and multilayer diode structures, the onset of the EL has been shown to be governed by the gradual increase of interfacial charges and the concomitant redistribution of the electrical field. ${ }^{9,15}$ It is often admitted that at $t>t_{d}$ the EL signal consists of two exponential components which are related to the transport of the charge carriers in the bulk. ${ }^{4,7}$ In other studies, the EL decay with long tails in the milliseconds scale has been reported and interpreted in terms of a bimolecular Langevintype electron-hole recombination in which the decay time is modulated by the majority carrier (hole) density. ${ }^{14}$

Modeling the EL transient patterns is widely recognized

\footnotetext{
${ }^{\text {a) }}$ Author to whom the correspondence should be addressed; electronic email: garciag@uji.es
}

as a complex issue in which fundamental knowledge about charge carrier dynamics (injection, transport, and recombination) and on excited energy (exciton) kinetics is needed. ${ }^{5-7,11}$ When a bias is applied to an OLED independent charge carriers are injected from the electrodes and drift to reach each other in the recombination zone. Upon reaching each other, opposite sign carriers can form excitons, correlated charge pairs, or remain as independent charges. Generally, the dynamics of exciton formation is much faster than that for independent charge recombination. Under these conditions it is expected that the EL transients are governed by the current evolution, at least after the initial few nanoseconds. ${ }^{16}$ Nevertheless, in some cases an EL decay time much longer than the switching of the current has also been reported. ${ }^{14}$

The observation of long tails in the fluorescence decay ${ }^{17}$ and a delayed luminescence ${ }^{18,19}$ provide evidence for the existence of long-lived excited energy states in poly(phenylene vinylene) (PPV). The importance of these factors on the overall luminescence yield is not well understood. Several photoluminescence studies of PPV derivatives have addressed the issue of the long-lived (milliseconds to microseconds) emission due to delayed exciton kinetics. For instance, triplet-triplet annihilation in singlet excitons was observed to yield lifetimes as long as $200 \mu \mathrm{s},{ }^{18}$ alerting on the importance of understanding triplet dynamics as they are the most prolific of energy states generated by charge carrier recombination. Photoinduced modulated-spectroscopy analyses have also provided evidence for triplet kinetics with lifetimes around $450 \mu \mathrm{s} .{ }^{20}$ The role of long-lived dark states acting as intermediate states in the energy relaxation of phenylene vinylene polymers was also proposed. ${ }^{21}$

The specific aim of this work was the analysis of the current and the EL transient responses of standard PPV-based 


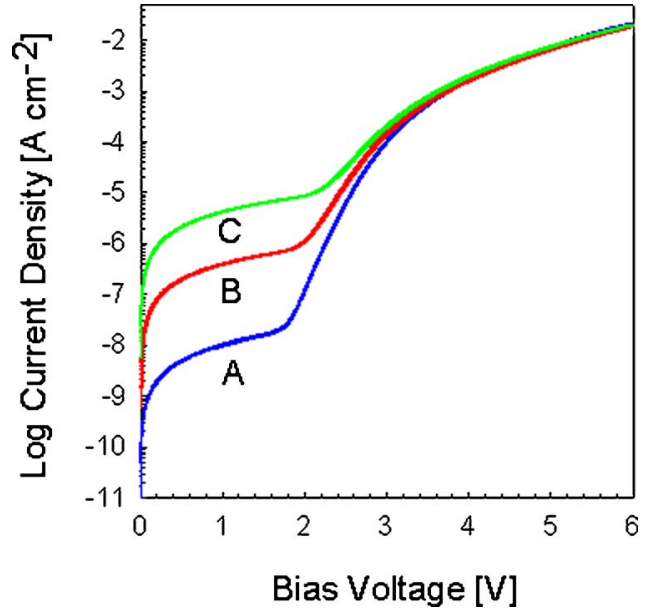

FIG. 1. (Color online) Example of $J-V$ characteristics of OLEDs with structures ITO/PEDOT:PSS/SY/Ba with different device active areas: A $\left(6.03 \mathrm{~cm}^{2}\right)$, B $\left(0.11 \mathrm{~cm}^{2}\right)$, and $\mathrm{C}\left(0.01 \mathrm{~cm}^{2}\right)$.

light-emitting devices to an applied bias pulse. In our experiments, the EL time response is longer (milliseconds scale) than the current decay time by more than one order of magnitude when using small area devices $\left(<0.1 \mathrm{~cm}^{2}\right)$. For larger device areas $\left(\sim 6 \mathrm{~cm}^{2}\right)$ the rapid current decay limits the EL decay as the bias voltage is increased. The results are interpreted either in terms of a Langevin-type, bimolecular recombination kinetics governed by the minority carriers (electrons), or caused by the slow release of trapped charges, presumably electrons.

\section{EXPERIMENT}

In this study we have used a PPV copolymer, "super yellow" (SY) prepared by Merck OLED Materials GmbH, as the light-emitting polymer. ${ }^{22}$ Standard device layouts were prepared, using a $200 \mathrm{~nm}$ thick [poly(ethylenethioxythiophene):poly(styrenesulfonic) acid, PEDOT:PSS] hole injection layer, and a $100 \mathrm{~nm}$ thick SY layer which was covered with a $10 \mathrm{~nm}$ thick layer of barium. An additional $100 \mathrm{~nm}$ thick Al layer was evaporated on top of the cathode metal to protect the lower work function metals and serve as an optical mirror. Details on device preparation were published elsewere. ${ }^{23} \mathrm{~J}-\mathrm{V}$ characteristics were collected using an AutoLab PGSTAT30 equipment (Fig. 1). Steady-state electroluminescence values around $1700 \mathrm{~cd} \mathrm{~m}^{-2}$ were achieved at $6 \mathrm{~V}$ bias. The transient electroluminescence response to a voltage pulse was collected using a fast enough photodiode (Centronic OSD100-7, response time $6 \mu \mathrm{s}$ ) connected to a digital oscilloscope (Hewlet Packard Infinium 1 Gsample/s). Simultaneously the current transient was registered. Rise and fall transient times of the voltage steps were selected to be smaller than $40 \mu$ s in order to avoid instrumentation delays. Devices with three different active areas were tested: A $\left(6.03 \mathrm{~cm}^{2}\right)$, B $\left(0.11 \mathrm{~cm}^{2}\right)$, and $\mathrm{C}\left(0.01 \mathrm{~cm}^{2}\right)$. We made sure that the $R C$ time constant of the setup did not affect the EL and the current transient signal shapes.

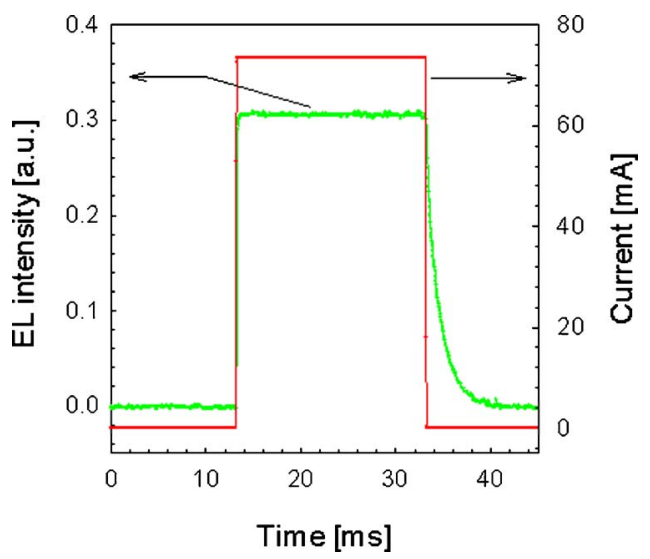

FIG. 2. (Color online) Example of current and EL transients driving by a bias voltage pulse between 0 and $6 \mathrm{~V}$ : Device A $\left(6.03 \mathrm{~cm}^{2}\right)$.

\section{RESULTS}

A typical transient response, for both the current and the EL, to a rectangular voltage pulse is shown in Fig. 2. One can observe that the current decay is extremely fast (microseconds scale) in comparison with the EL transient, which exhibits a long tail in the milliseconds scale. This pattern is observed for different voltage steps and device areas. It indicates that the current and the EL kinetics might be considered as decoupled in these devices. A close examination of the EL transients reveals that the resulting rise (fall) time is determined by the voltage of the top (bottom) value of the rectangular voltage pulse. Moreover, a similar time constant of the EL transients is encountered irrespective of the polarity of the initial voltage step. This simplifies the analysis allowing us to focus exclusively on the decay responses (negative voltage slopes). Figure 3(a) shows the normalized EL decays for different low bias values and a constant initial voltage level $(6 \mathrm{~V})$ using device A. As observed, the straightline decay in a semilogaritmic representation points to the occurrence of a simple first-order kinetics for the radiative process.

Figure 4 summarizes the EL response time as a function of the bias voltage for devices with different active areas. By examining Fig. 4 one can observe that all devices exhibit similar values $(\sim 1.45 \mathrm{~ms})$ for step low voltages in the range of $0-3 \mathrm{~V}$, independent of the device area. The EL response time for large area devices shows a transition between two values. The decay kinetics of transitions toward higher bias voltage levels produces relatively faster responses $(112 \mu \mathrm{s})$ than those encountered for low voltage levels. This is not observed for small area devices for which the characteristic response time shows a rather constant value around $1.50 \mathrm{~ms}$. Device B shows the onset in the response-time decrement around $5 \mathrm{~V}$.

In order to deeply investigate the possible relation between the charge carriers and the EL signal, the current decays are shown on a semilogaritmic scale in Fig. 3(b). As pointed out previously, there is a fast current fall of more than two orders of magnitude (response time $\tau_{1} \approx 100 \mu \mathrm{s}$ ) followed by a slower decay, $\tau_{2}$ in the milliseconds scale. The slow current transits are characterized by a transition around $4 \mathrm{~V}$ between decay to recover behavior (after a current over- 
(a)

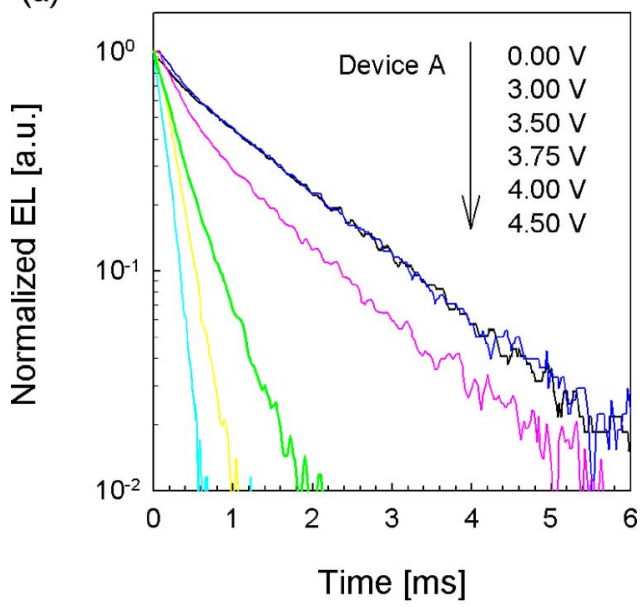

(b)

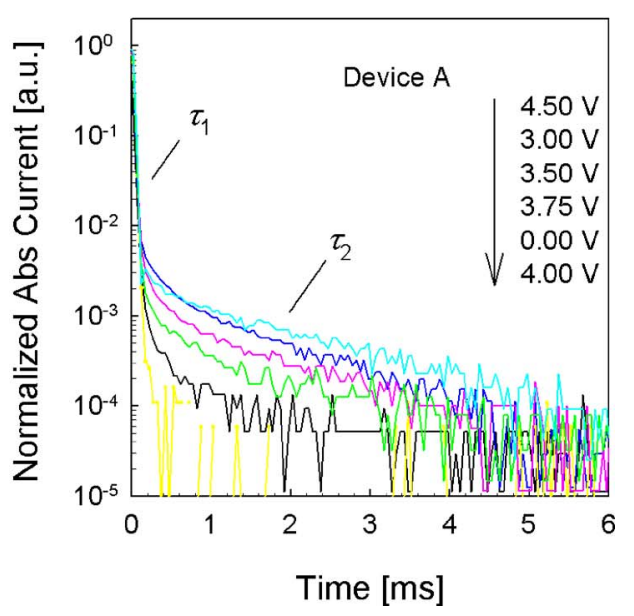

FIG. 3. (Color online) (a) Normalized electroluminescence decay for bias voltage step between $6 \mathrm{~V}$ and different values marked in the figure (device A). Slow transients correspond to large steps. Decays are approximately exponentials. (b) Normalized current decay for bias voltage step between $6 \mathrm{~V}$ and different values marked in the figure (device A). Two response times are observed: a fast decay $\tau_{1} \approx 100 \mu \mathrm{s}$, which represents more than two orders of magnitude in the current switch, and a slow kinetics $\tau_{2}$. A current overshoot appears for final voltages higher than $4 \mathrm{~V}$.

shoot). This explains the minimum exhibited by $\tau_{2}$. By comparing the EL and the current transient one can conclude that the fast response of the current $\tau_{1}$ is, in fact, limiting the EL decay when high-voltage steps are applied and large area devices are used (see Fig. 4). One might argue that the slow current response time $\tau_{2}$ has a direct influence on the EL decay. A close examination of the different response times in Fig. 4 shows that EL decay time $1.45 \mathrm{~ms}$ for low final bias (device A) lies within the same order of magnitude as that encountered for $\tau_{2}$. It seems possible therefore that the underlying mechanisms governing the response time of the current are related to the EL decay occurring in the milliseconds scale. Therefore, the slow current dynamics, with its specific response time $\tau_{2}$, would be ascribed to mechanisms involved in the emission process and delayed carrier motion.

\section{DISCUSSION}

When a bias is applied to an OLED, independent carriers are injected from the electrodes and drift to the recombina-

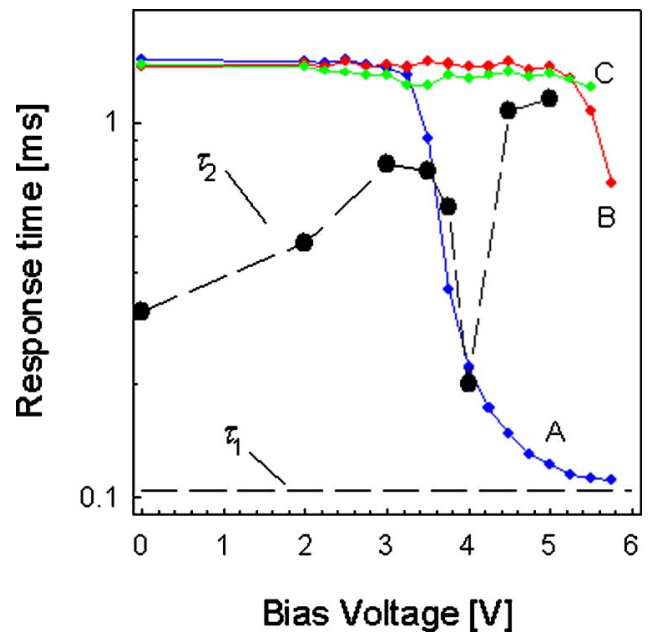

FIG. 4. (Color online) EL response time extracted from fits using Eq. (2) for three different devices using bias steps between $6 \mathrm{~V}$ and different final voltages. Fast $\tau_{1}$ and slow $\tau_{2}$ current response time are also marked.

tion zone. After reaching the steady state, the carriers can either form excitons and correlated charge pairs, or remain as independent carriers. As emitting organic materials are generally insulators, the currents are space-charge limited. The evaluation of the dynamics of the independent carriers is modeled by the combination of the continuity and the Poisson equations. In principle there are two different limiting cases suitable for analyzing our results. Either the exciton or correlated charge pairs kinetics (characterized by the exciton lifetime $\tau_{\mathrm{ex}}$ ) is slower than the charge carrier dynamics (characterized by $\tau_{1}$ and $\tau_{2}$ as described above), $\tau_{\mathrm{ex}} \gg \tau_{2}$; or on the contrary, exciton kinetics is invisible, $\tau_{\mathrm{ex}} \ll \tau_{1}$. This last case simply means that the EL signal monitors the recombination mechanisms within the complex carrier dynamics.

\section{A. Exciton kinetics}

It is interesting to point out that the experimentally observed exponential EL decay behavior can originate from a simple exciton relaxation. This approach then assumes that $\tau_{\mathrm{ex}} \gg \tau_{2}$. Let us consider the continuity equation for the exciton density $S(x, t)$, which includes the generation, diffusion, and decay terms as

$$
\frac{d S(x, t)}{d t}=\gamma R(x, t) n(x, t) p(x, t)+D_{s} \frac{d^{2} S(x, t)}{d x^{2}}-\frac{S(x, t)}{\tau_{\mathrm{ex}}} .
$$

The first term corresponds to exciton generation resulting from the bipolar recombination of electrons $n$ and holes $p$ with $R$ as a recombination rate coefficient. Here $\gamma=1 / 4$ from a simple spin statistics point of view. The second summand assumes a Fickian diffusion mechanism for excitons in which $D_{s}$ is the diffusion coefficient. The last term stands for the exciton decay with $\tau_{\mathrm{ex}}$ as the exciton lifetime. The diffusion coefficient and the exciton lifetime determine its diffusion length as $D_{s}=l^{2} / \tau_{\text {ex }}$. The transient experiments are interpreted in the following way: before the potential step application the generation term in Eq. (1) is assumed to have achieved the stationary initial value $S_{0}(x, t=0)$. By comparing typical time scales involved in both the current and the EL transients, one can infer that free carrier densities reach 
the steady state at times shorter than those characteristic for the EL response. The generation term in Eq. (1) can then be considered as a constant in the stationary final value $S_{1}(x, t$ $\rightarrow \infty)$. It is also widely accepted that the diffusion length for excitons lies within the range of 4-7 nm. ${ }^{24}$ This would entail that the exciton decay occurs near the generation position so as to neglect the diffusion term in a first approximation. Under these assumptions, Eq. (1) is reduced to a simple firstorder kinetics in good agreement with our experimental observations of Fig. 3(a). The decay part of the pulsed response obeys the following expression:

$$
\begin{aligned}
S(x, t)= & {\left[S_{0}(x, 0)-S_{1}(x, t \rightarrow \infty)\right] \exp \left(-t / \tau_{\mathrm{ex}}\right) } \\
& +S_{1}(x, t \rightarrow \infty) .
\end{aligned}
$$

Despite the reported observations concerning the existence of long-lived excited states in PPV-related materials, ${ }^{17-21}$ this first case $\left(\tau_{\mathrm{ex}} \gg \tau_{2}\right)$ is difficult to imagine because the exciton and coupled charged pairs decay times are usually much faster than the charge carrier dynamics. ${ }^{16}$

\section{B. Bimolecular radiative recombination}

The second case (i.e., $\tau_{\mathrm{ex}} \ll \tau_{1}$ ) is in our opinion representing the phenomena observed in the light-emitting device. However, one question remains: How can these long exponential EL decays be related to the nontrivial charge carrier dynamics? A sound response to this question should involve the transient analysis of a model for the complete device including charge transport, recombination, and injection terms. As this lies beyond the scope of this work, instead we will provide some plausible arguments that reinforce the idea that an electron-limited injecting cathode is the origin of the observed monomolecularlike EL decay.

To model the response of our devices we included the following dynamic equations for electrons and holes:

$$
\begin{aligned}
& \frac{d n}{d t}=\mu_{e} \frac{d(n E)}{d x}-R n p, \\
& \frac{d p}{d t}=-\mu_{h} \frac{d(p E)}{d x}-R n p,
\end{aligned}
$$

where $E$ is the position-dependent electrical field. $\mu_{e}$ and $\mu_{h}$ denote the electron and hole mobilities, which for the sake of simplicity we will consider as field independent. Diffusion currents and trapping dynamics have been neglected in a first approximation. The equation system is completed by recalling the Poisson's equation,

$$
\frac{d E}{d x}=\frac{e}{\varepsilon}(p-n) .
$$

Here $e$ is the elementary charge and $\varepsilon$ stands for the dielectric constant of the organic material. Equations (3)-(5) can be solved numerically to get the steady-state carrier densities, and recombination term. By using typical values for PPV-derivative parameters ${ }^{25}\left(\mu_{h}=5 \times 10^{-7} \mathrm{~cm}^{2} \mathrm{~V}^{-1} \mathrm{~s}^{-1}, \mu_{e}\right.$ $\left.=5 \times 10^{-9} \mathrm{~cm}^{2} \mathrm{~V}^{-1} \mathrm{~s}^{-1}, \varepsilon=3 \varepsilon_{0}\right)$ and assuming the recombination constant being determined by the Langevin term,
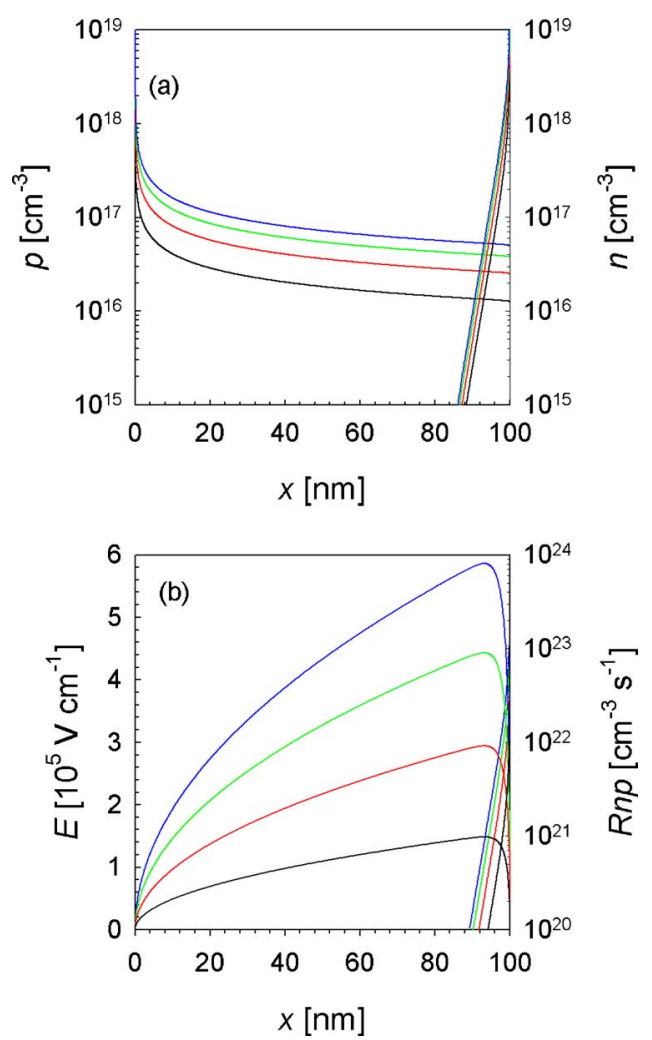

FIG. 5. (Color online) Results of the device simulation considering both anode and cathode as Ohmic contacts. (a) Carrier density as a function of the position for different values of the driving voltage $V=V_{\text {bias }}-V_{\mathrm{BI}}$ equal to $4,3,2$, and $1 \mathrm{~V}$ (top to down). (b) Electrical field and recombination parameter $R$ using the same driving voltages. Simulation parameters: $\mu_{h}=5$ $\times 10^{-7} \mathrm{~cm}^{2} \mathrm{~V}^{-1} \mathrm{~s}^{-1}, \mu_{e}=5 \times 10^{-9} \mathrm{~cm}^{2} \mathrm{~V}^{-1} \mathrm{~s}^{-1}, \varepsilon=3 \varepsilon_{0}$, and $L=100 \mathrm{~nm}$.

$$
R=\frac{e}{\varepsilon}\left(\mu_{e}+\mu_{h}\right)
$$

one obtains the results shown in Fig. 5. In this case we have regarded both anode $(x=0)$ and cathode $(x=L)$ as Ohmic contacts. This simulation is performed in such a way that the dc currents shown in Fig. 1 are achieved for a driving voltage $V=V_{\text {bias }}-V_{\mathrm{BI}}\left(V_{\mathrm{BI}}\right.$ denotes the built-in potential), and a $L=100 \mathrm{~nm}$ thick active polymer layer. As expected, charge carrier distributions are observed typical for space-charge limited currents. As a consequence of the pronounced difference in the mobility values, electrons are only able to penetrate into a thin layer of the organic film adjacent to the cathode, while holes transfer the whole organic layer and reach the opposite contact. The transients of current depicted in Fig. 3(b) would correspond to transitions between two of these steady-state carrier-density profiles. The recombination current is directly related to the integral of the carrier density product,

$$
J_{r}=e \int_{0}^{L} R n p d x .
$$

By examining Fig. 5(b), one realizes that the electrical field finds a maximum in the vicinity of the recombination zone. However, it is rather improbable that such a carrier density change could give rise to the simple exponential decay as observed by transient EL, because the recombination term 
contains the product $n p$, with both densities varying as a function of time and position. Moreover, this simple simulation entails that the dc current is mainly determined by holes at the anode $\left[J=e \mu_{h} p(0) E(0)\right]$ and electrons at the cathode $\left[J=e \mu_{e} n(L) E(L)\right]$ because of the Ohmic character of such contacts, $p(0) \gg n(0)$, and similarly $n(L) \gg p(L)$. Since more than $99 \%$ of the current step falls abruptly in the initial decay $\tau_{1} \approx 100 \mu \mathrm{s}$, there is a rapid decrease of both hole and electron densities at the respective external contacts. This would imply that the relaxation of the carriers inside the sample occurs mainly due to drift currents. Transit times can be obtained readily for different values of the driving voltage and always result in values less than $100 \mu$ s for the parameters used in the simulation. This suggests that our observations are hardly understandable assuming Ohmic contacts in both electrodes and free carrier recombination.

The partial Ohmic character of the cathode has been outlined previously. ${ }^{23,26,27}$ It is thus reasonable to investigate the effect of a limitation in the electron injection. The injection properties of metal-organic interfaces are believed to depend on the features of a thin layer contacting the metal. ${ }^{28,29}$ This is particularly true for metal-on-organic depositions which form structurally and chemically more complex interfaces than their organic-on-metal counterparts. ${ }^{30}$ Isolated metal atoms evaporated on an organic surface are chemically more active and capable of diffusing more deeply into the polymer matrix. Diffusion and chemical reactions can lead to the formation of extended regions comprising new metal-organic complexes with a different electronic structure than that observed in the bulk of the organic film. As a working hypothesis, we assume that the available electron accepting states in the first organic layers is reduced with respect to the lowest unoccupied molecular orbital (LUMO) bulk density. This approach highlights the inability of the voltage to modulate the electron density at the organic layer contacting the electrode metal. Strictly speaking, the metal contact can be considered as Ohmic in the sense that it is able to provide the required electron current but limited by the effect of the reduced interfacial electron density of states. To account for this effect in the numerical simulation the bordering conditions need to be adjusted, keeping the anode contact Ohmic (unlimited hole injection) but fixing the density of electrons available at the cathode for injection into the organic film. Results of the simulation are shown in Fig. 6 for a fixed value of the density of electrons $n(L)=2.5 \times 10^{16} \mathrm{~cm}^{-3}$. All other parameters were used unaltered. The driving potential is distributed within the organic layer according to Eqs. (3)-(5). By examining Fig. 6, one can observe that the hole density at the cathode exceeds the electron density, as expected for an injection-limited cathode. Surprisingly, the electron density profile does not change with the driving voltage, which allows regarding $n$ as a time-independent parameter. Equation (4) can be rewritten as

$$
\frac{1}{R n} \frac{d(e R n p)}{d t}=-e \mu_{h} \frac{d(p E)}{d x}-e R n p .
$$

The integration over the active device thickness gives the following expression taking into account Eq. (7):
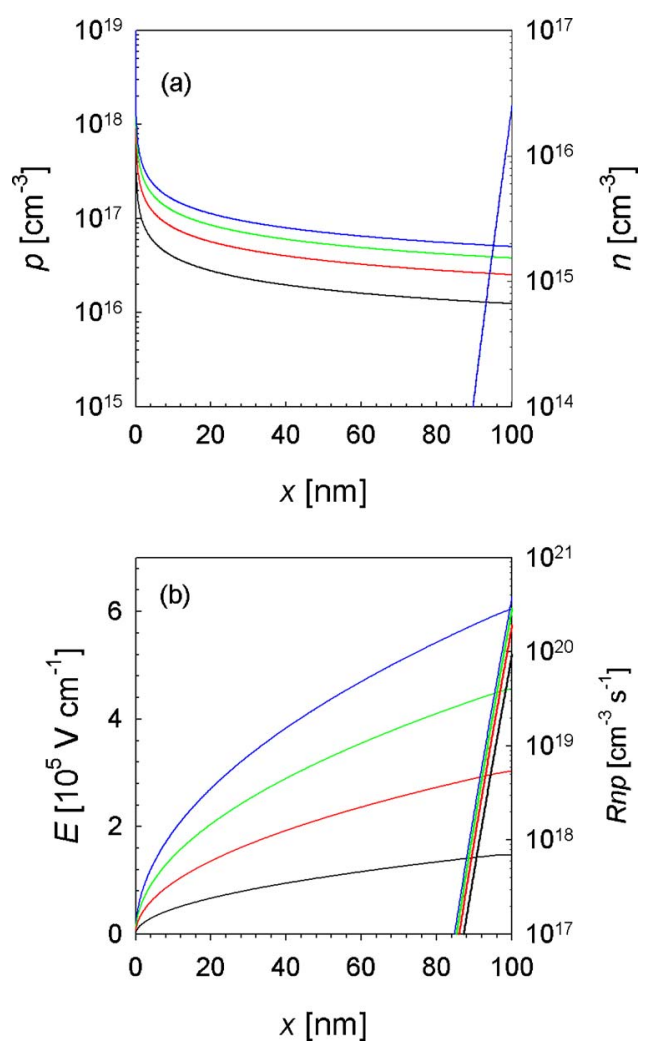

FIG. 6. (Color online) Results of the device simulation considering solely anode as Ohmic contact. The electron density at the cathode has been kept fixed at $n(L)=2.5 \times 10^{16} \mathrm{~cm}^{-3}$. (a) Carrier density as a function of the position for different values of the driving voltage $V=V_{\text {bias }}-V_{\mathrm{BI}}$ equal to $4,3,2$, and $1 \mathrm{~V}$ (top to down). (b) Electrical field and recombination parameter $R$ using the same driving voltages. Simulation parameters: $\mu_{h}=5$ $\times 10^{-7} \mathrm{~cm}^{2} \mathrm{~V}^{-1} \mathrm{~s}^{-1}, \mu_{e}=5 \times 10^{-9} \mathrm{~cm}^{2} \mathrm{~V}^{-1} \mathrm{~s}^{-1}, \varepsilon=3 \varepsilon_{0}$, and $L=100 \mathrm{~nm}$.

$$
\frac{1}{R} \int_{0}^{L} \frac{1}{n} \frac{d(e R n p)}{d t} d x=-e \mu_{h} \int_{0}^{L} \frac{d(p E)}{d x} d x-J_{r} .
$$

Since the time derivative affects the hole but not the electron density, it is possible to approximate the left side of the previous expression by extracting the average electron density $\bar{n}$ in such away that

$$
\frac{1}{R \bar{n}} \frac{d J_{r}}{d t} \approx e \mu_{h}[p(0) E(0)-p(L) E(L)]-J_{r}
$$

This last expression represents a decay of the EL which exhibits an exponential form provided the the hole currents at both contacts have reached the steady-state value. One can interpret that a portion of the holes relaxes within the recombination zone whereas the main part drifts toward the electrodes and simply escapes. The integration of Eq. (10) yields an expression similar to Eq. (2),

$$
J_{r}(t)=\left[J_{r}(0)-J_{r}(t \rightarrow \infty)\right] \exp (-t / \tau)+J_{r}(t \rightarrow \infty) .
$$

In this expression the time constant is

$$
\tau=\frac{1}{R \bar{n}},
$$

which is essentially voltage independent.

The steady-state recombination current at each driving voltage equals the difference in hole current at both elec- 
trodes. From this point of view, the fast current decay $\left(\tau_{1}\right.$ $\approx 100 \mu \mathrm{s}$ ) should be related to a rapid carrier (hole) extraction and the corresponding reaching of the steady-state current. Residual holes in the recombination zone disappear slowly by the electron-determined recombination rate. It should be stressed that the previous derivation of Eq. (11) includes all the contributions involved in the recombination process. It is well known that an important part of the excitons are quenched near the cathode and therefore they do not contribute to the light emission. This means that the average electron density $\bar{n}$ introduced in the approximation done in Eq. (10) should be linked with a narrower layer close to the quenching zone; this is because the electron density profile also drops rapidly towards the anode.

By regarding the cathode as an injection limiting contact, we are able to explain one of the more important features of the EL transients: for small area devices the decay time is almost constant and independent of the bias voltage. This fact can be easily explained by noting that the time constant is proportional to the average electron density $\bar{n}$, which is in turn voltage independent as shown in Fig. 6. Long EL decay tails are characterized by a nearly constant decay time $\tau \approx 1.45 \mathrm{~ms}$, using the recombination constant of Eq. (6), $R=3.0 \times 10^{-13} \mathrm{~cm}^{3} \mathrm{~s}^{-1}$, electron density at the thin recombination zone results in $\bar{n} \approx 2.3 \times 10^{15} \mathrm{~cm}^{-3}$, which is reasonable value taking into account the electron density profile depicted in Fig. 6(a). One might argue that it is also possible that the recombination time is related to the average hole density near the cathode so as to give $1 / \tau=R \bar{p}$. In fact, $p$ exhibits a flat profile and it is almost position independent at the recombination zone [see Fig. 6(a)]. But in such a case $\tau$ would decrease as a function of the voltage. This is in contradiction with the time response observed for small area devices which show a nearly constant time constant. Hence this argument can be discarded.

\section{Trapped charges}

Despite the plausible interpretation of the EL transients presented above, it must also be stressed that the dynamics of trapped charges in the recombination zone can be the origin of our observations. Cheon and Shinar ${ }^{16}$ recently related the EL decay tail registered after device turn off $(<100 \mu \mathrm{s})$ to the recombination of initially uncorrelated trapped charges. This approach interprets the decay time $\tau$ as a measurement of the release rate from traps. Although we cannot completely discard this approach we feel it is not the correct explanation due to following arguments. It should be noted that the exponential EL decay starts precisely from the value reached by the EL steady-state signal (see Fig. 2), what would indicate that the recombination mechanism in PPVbased OLED devices is always occurring via trapped charges with no contribution from direct free electron-hole recombination. Secondly, the exponential decay exhibited by the EL transients implies a monoenergetic trap level. This is unlikely as electron trapping sites are distributed in energy such that they yield power-law dependences with time.

\section{Effect of device area}

Finally, some comments are needed to understand the difference between the small versus large area devices. It is evident by examining Fig. 1 that the current density is independent of the device area for usual operating bias. It is then a natural consequence to assume a one dimensional (1D) device model in order to interpret the results. However, the area dependence encountered for the EL transient time is quite surprising and hardly understandable by using simple $1 \mathrm{D}$ pictures. The area effect points to an electron injection mechanism dependent on the device position. One simple way of explaining such a behavior is to consider a difference in electron injection between the perimeter and the center regions of the device. To distinguish between perimeter and bulk mechanisms is a common practice in the analysis of current-voltage characteristics of GaAs diodes and solar cells. ${ }^{31}$ In those devices there is evidence for the different role played by the perimeter in contrast to the bulk recombination processes. ${ }^{32}$ In the case of OLEDs, the structure of the luminescent polymer located near the device perimeter shows certain particularities in comparison with true "bulk" regions, completely covered by the cathode metal. As the device area is enlarged central regions play a more significant role with respect to the perimeter ones. It should be noted that such a perimeter effect does not affect the hole injection, presumably because of the Ohmic character of the anode. Since the principal part of the current is controlled by holes this explains why the $J-V$ curves are independent of the device area as observed in Fig. 1 for voltages grater than $V_{\mathrm{BI}}$.

Another explanation for the different EL transients observed for OLED devices with different active areas is related to a temperature effect. It is well known that large-area devices have lower ability to dissipate the heat produced during device operation. A higher operation temperature would influence the electron injection and thereby the EL transient response. Additionally, considering the trapped charge approach, one can imagine that charges are easier released from trapping centers at elevated temperatures. Changes in the detrapping time from 1450 down to $112 \mu$ s (as exhibited by device A) would imply activation energies of such process around $2 \mathrm{eV}$ for a temperature increment of $10 \mathrm{~K}$. These activation energies correspond to deep electron traps. We will focus on these effects in future works.

\section{CONCLUSIONS}

We have demonstrated that the observed long exponential EL tails in PPV-based OLEDs can be explained by assuming a device model in which the electron injection contact is limiting. In such conditions, the steady-state solution of the equation system governing the device operation yields an electron density profile, which is voltage independent and located at the vicinity of the cathode. The EL originates from a Langevin-type, bimolecular free charge recombination, whereas the charge recombination is governed by the minority carriers (electrons), or modulated by the release of trapped charges, presumably electrons. The device current is mainly determined by the transport of the fastest carriers (holes). 


\section{ACKNOWLEDGMENTS}

The authors would like to thank Merck OLED Materials for the supply of the light-emitting polymer SY. The work was supported by the Spanish Ministry of Education and Science (MAT2004-038499), Generalitat Valenciana, and Fundació Caixa Castelló Bancaixa under Project No. P1 1B2005-12. One of the authors (H.B.) acknowledges the support of the Program "Ramon y Cajal" of the Spanish Ministry of Education and Science.

${ }^{1}$ S. Karg, V. Dyakonov, M. Meir, W. Riess, and G. Paasch, Synth. Met. 67, 165 (1994).

${ }^{2}$ P. E. Burrows, Z. Shen, V. Bulovic, D. M. McCarty, S. R. Forrest, J. A. Cronin, and M. E. Thompson, J. Appl. Phys. 79, 7991 (1996).

${ }^{3}$ H. Becker, A. Lux, A. B. Holmes, and R. H. Friend, Synth. Met. 85, 1289 (1997).

${ }^{4}$ J. Pommerehne, H. Vestweber, Y. H. Tak, and H. Bässler, Synth. Met. 76, 67 (1996)

${ }^{5}$ V. R. Nikitenko, V. I. Arkhipov, Y. H. Tak, J. Pommerehne, H. Bässler, and H.-H. Hörhold, J. Appl. Phys. 81, 7514 (1997).

${ }^{6}$ V. R. Nikitenko, Y. H. Tak, and H. Bässler, J. Appl. Phys. 84, 2334 (1998).

${ }^{7}$ D. J. Pinner, R. H. Friend, and N. Tessler, J. Appl. Phys. 86, 5116 (1999)

${ }^{8}$ V. Savvateev, A. Yakimov, and D. Davidov, Adv. Mater. (Weinheim, Ger.) 11, 519 (1999).

${ }^{9}$ S. Barth, P. Müller, H. Riel, P. F. Seidler, W. Riess, H. Vestweber, and H. Bässler, J. Appl. Phys. 89, 3711 (2001).

${ }^{10}$ J. M. Lupton, V. R. Nikitenko, I. D. W. Samuel, and H. Bässler, J. Appl. Phys. 89, 311 (2001).

${ }^{11}$ B. Ruhstaller, S. A. Carter, S. Barth, H. Riel, W. Riess, and J. C. Scott, J. Appl. Phys. 89, 4575 (2001).

${ }^{12}$ M. Ichikawa, J. Amagai, Y. Horiba, T. Koyama, and Y. Taniguchi, J. Appl. Phys. 94, 7796 (2003).

${ }^{13}$ J.-S. Kim, H. Kajii, and Y. Ohmori, Thin Solid Films 499, 343 (2006).
${ }^{14}$ A. Rihani, L. Hassine, J.-L. Fave, and H. Bouchriha, Org. Electron. 7, 1 (2006).

${ }^{15}$ W. Brütting, H. Riel, T. Beierlein, and W. Riess, J. Appl. Phys. 89, 1704 (2001).

${ }^{16}$ K. O. Cheon and J. Shinar, Phys. Rev. B 69, 201306(R) (2004).

${ }^{17}$ L. J. Rothberg, M. Yan, F. Papadimitrakopoulos, M. E. Galvin, and E. W. Kwock, Synth. Met. 80, 41 (1996).

${ }^{18}$ J. Partee, E. L. Frankevich, B. Uhlhron, J. Shinar, Y. Ding, and T. J. Barton, Phys. Rev. Lett. 82, 3673 (1999).

${ }^{19}$ V. Dyakonov and E. Frankevich, Chem. Phys. 227, 203 (1998).

${ }^{20}$ E. Gershman, T. Drori, C. Herzog, Y. Eichen, and E. Ehrenfreund, Synth. Met. 1545, 237 (2005).

${ }^{21}$ S.-H. Lim, T. G. Bjorklund, K. M. Gaab, and C. J. Bardeen, J. Chem. Phys. 117, 454 (2002).

${ }^{22}$ H. Becker, H. Spreitzer, W. Kreuder, E. Kluge, H. Schenk, I. Parker, and Y. Cao, Adv. Mater. (Weinheim, Ger.) 12, 42 (2000).

${ }^{23}$ J. Bisquert, G. Garcia-Belmonte, A. Pitarch, and H. J. Bolink, Chem. Phys. Lett. 422, 184 (2006).

${ }^{24}$ D. E. Markov, C. Tanase, P. W. M. Blom, and J. Wildeman, Phys. Rev. B 72, 045217 (2005).

${ }^{25}$ L. Bozano, A. Carter, J. C. Scott, G. G. Malliaras, and P. J. Brock, Appl. Phys. Lett. 74, 1132 (1999).

${ }^{26}$ V. I. Arkhipov, H. von Seggern, and E. V. Emelianova, Appl. Phys. Lett. 83, 5074 (2003)

${ }^{27}$ G. Garcia-Belmonte, H. J. Bolink, and J. Bisquert, Phys. Rev. B 75, 085316 (2007).

${ }^{28}$ Y. Shen, A. R. Hosseini, M. H. Wong, and G. G. Malliaras, ChemPhysChem 5, 16 (2004).

${ }^{29}$ C. Shen and A. Kahn, Org. Electron. 2, 89 (2001).

${ }^{30}$ C. Shen, A. Kahn, and I. Hill, in Conjugated Polymer and Molecular Interfaces, 1st ed., edited by W. R. Salaneck, K. Seki, A. Kahn, and J.-J. Pireaux (Marcel Dekker, New York, 2002).

${ }^{31}$ P. E. Dodd, T. B. Stellwag, R. Melloch, and M. S. Lundstrom, IEEE Trans. Electron Devices 38, 1253 (1991).

${ }^{32}$ A. Belghachi and S. Khelifi, Sol. Energy Mater. Sol. Cells 90, 1 (2006). 